\title{
Article
}

\section{Rapid interrogation of the physical and chemical characteristics of salbutamol sulphate aerosol from a pressurised metered-dose inhaler (pMDI)}

Tong, H.-J., Fitzgerald, C., Gallimore, P. J., Kalberer, M., Kuimova, M. K., Seville, P. C., Ward, A. D. and Pope, F. D.

Available at http://clok.uclan.ac.uk/13613/

Tong, H.-J., Fitzgerald, C., Gallimore, P. J., Kalberer, M., Kuimova, M. K., Seville, P. C. ORCID: 0000-0001-8546-3474, Ward, A. D. and Pope, F. D. (2014) Rapid interrogation of the physical and chemical characteristics of salbutamol sulphate aerosol from a pressurised metered-dose inhaler (pMDI). Chemical Communications, 50 (98). pp. 15499-15502. ISSN 1359-7345

It is advisable to refer to the publisher's version if you intend to cite from the work. http://dx.doi.org/10.1039/C4CC05803H

For more information about UCLan's research in this area go to http://www.uclan.ac.uk/researchgroups/ and search for < name of research Group>.

For information about Research generally at UCLan please go to http://www.uclan.ac.uk/research/

All outputs in CLoK are protected by Intellectual Property Rights law, including Copyright law. Copyright, IPR and Moral Rights for the works on this site are retained by the individual authors and/or other copyright owners. Terms and conditions for use of this material are defined in the policies page. 


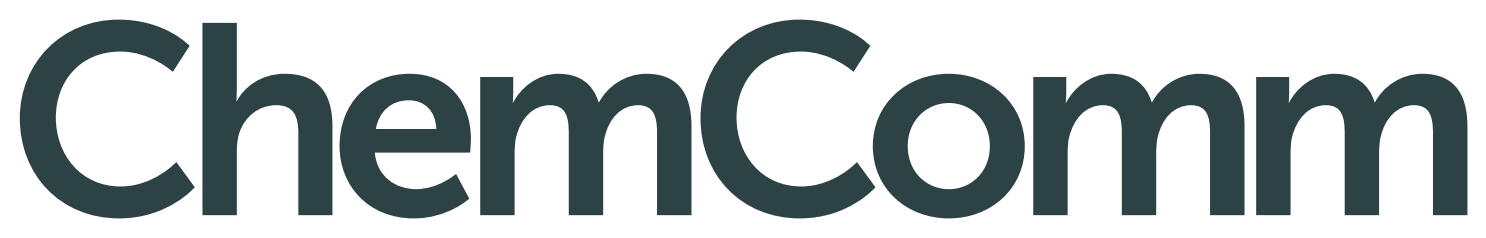

Chemical Communications

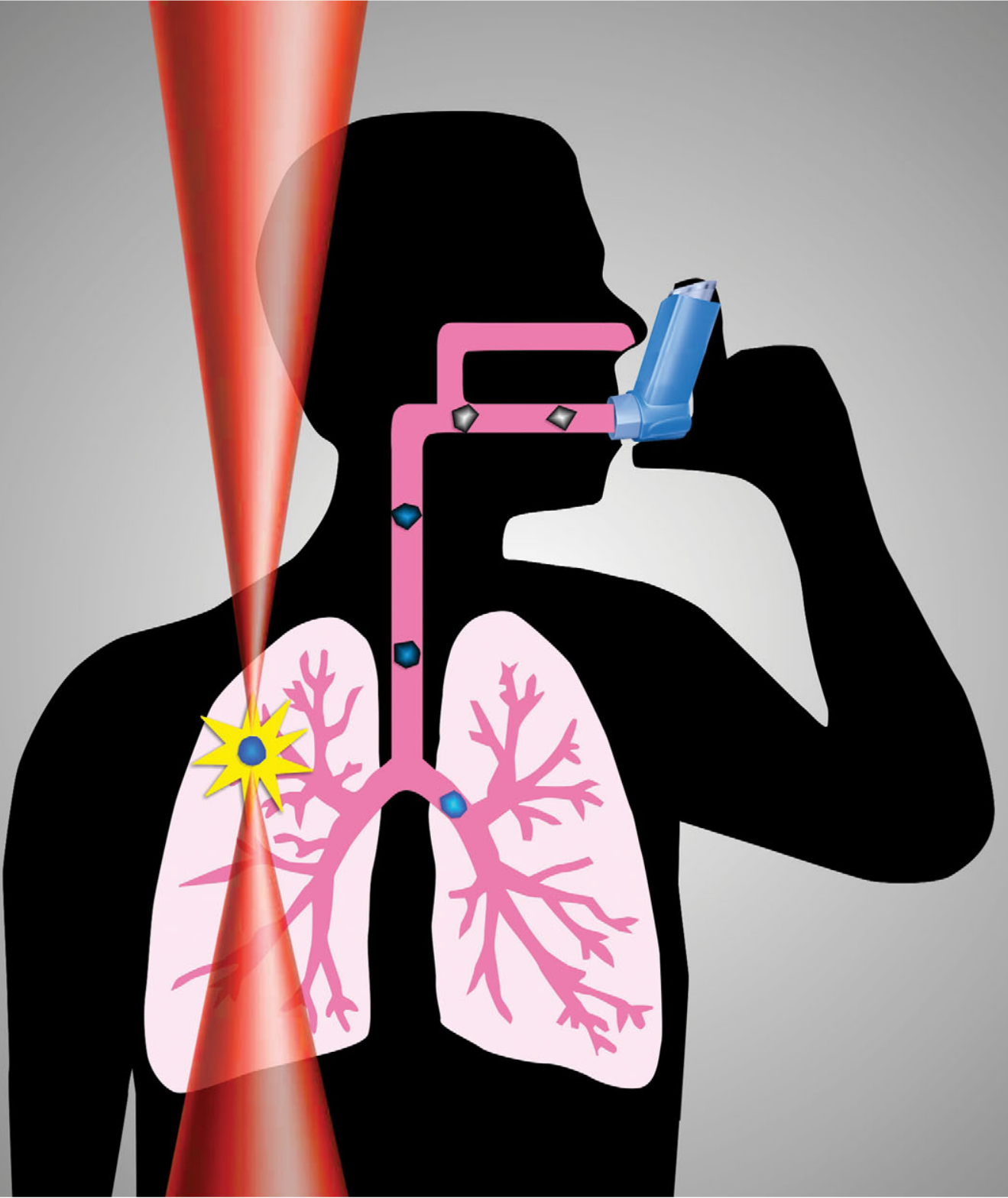

ISSN 1359-7345

\section{COMMUNICATION}

A. D. Ward, F. D. Pope et al.

Rapid interrogation of the physical and chemical characteristics of

salbutamol sulphate aerosol from a pressurised metered-dose

inhaler (pMDI)

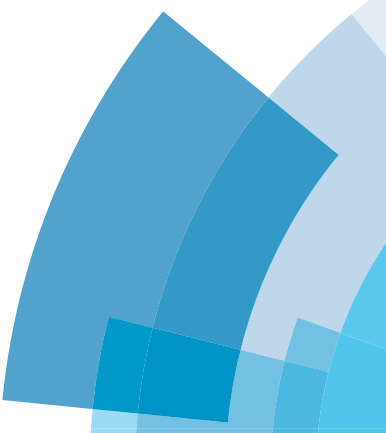




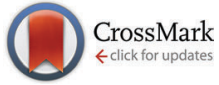

Cite this: Chem. Commun., 2014, 50, 15499

Received 25th July 2014,

Accepted 6th October 2014

DOI: $10.1039 / \mathrm{c} 4 \mathrm{cc} 05803 \mathrm{~h}$

www.rsc.org/chemcomm

Individual micron-sized solid particles from a Salamol ${ }^{\circledR}$ pharmaceutical inhaler are stably captured in air using an optical trap for the first time. Raman spectroscopy of the levitated particles allows online interrogation of composition and deliquescent phase change within a high humidity environment that mimics the particle's travel from inhaler to lung.

We investigate the physical and chemical evolution of the drug salbutamol sulphate (SS) from a Salamol ${ }^{\mathbb{R}}$ (manufacturer IVAX) pressurised metered-dose inhaler (pMDI) which contains a suspension of crystalline SS in 1,1,1,2-tetrafluoroethane (HFA-134a) propellant and minute amounts of ethanol. Upon actuation, SS is aerosolised generating micron sized particles. We compare the pMDI generated SS particles to bulk crystalline sample of SS and nebulised aqueous droplets of SS. This comparison permits the phase of the pMDI generated SS to be determined. The bulk crystalline sample and the nebulized aqueous solution of SS are used as reference systems.

An optical trap was used to probe the true aerosol phase of both the pMDI generated SS particles and the nebulised SS droplets. Optical trapping allowed for the stable levitation of the aerosol particles within a purpose built environmental chamber which mimicked the fast changing humidity encountered by pMDI-generated aerosol. Optical trapping achieves particle levitation by balancing the gravitational force, and the viscous drag created by air flow, by an optical force; ${ }^{1}$ it allows aerosols to be studied in a contact free manner and hence without the artefacts that contact with a surface can induce.

\footnotetext{
${ }^{a}$ Department of Chemistry, University of Cambridge, Lensfield Road, Cambridge, CB2 1EW, UK

${ }^{b}$ Department of Chemistry, Imperial College London, South Kensington Campus, London, SW7 2AZ, UK

${ }^{c}$ School of Pharmacy, University of Birmingham, Edgbaston, Birmingham, B15 2TT, UK

${ }^{d}$ Central Laser Facility, Rutherford Appleton Laboratory, Harwell, Oxford, OX11 OQX, UK. E-mail: andy.ward@stfc.ac.uk

${ }^{e}$ School of Geography, Earth and Environmental Sciences, University of

Birmingham, Edgbaston, Birmingham, B15 2TT,UK. E-mail:f.pope@bham.ac.uk

$\dagger$ Electronic supplementary information (ESI) available. See DOI: 10.1039/ $\mathrm{c} 4 \mathrm{cc} 05803 \mathrm{~h}$

\$ Now at the Max Planck Institute for Chemistry, Mainz, Germany.
}

Drugs are primarily delivered to the lung for the treatment of conditions local to the airway; for example anti-asthmatic drugs (e.g. salbutamol sulphate and beclometasone dipropionate). ${ }^{2}$ However, drug delivery to the lung is associated with a number of difficulties, including the relative inaccessibility of the lungs, necessitating the use of complex drug delivery devices to target the drug to the appropriate area of the airways.

The PMDI is presently the device of choice, accounting for around $70 \%$ of inhaler sales in the $\mathrm{UK}^{3}$ and is the most frequently used device for asthma and chronic obstructive pulmonary disease (COPD). ${ }^{4}$ These devices contain the particles of the drug suspended (or occasionally dissolved) in a pressurised gas, typically a hydrofluoroalkane propellant, along with relatively few other excipients (principally cosolvents such as ethanol or surfactants such as oleic acid). Actuating the device releases a metered volume of the suspension through an orifice contained within the pMDI housing; ${ }^{5}$ the released pressurised suspension undergoes rapid flash evaporation and phase expansion and forms inhalable solid aerosol particles.

The human respiratory tract efficiently filters atmospheric aerosols through the microphysics of impaction, sedimentation and diffusion. The size dependence of these competing processes results in particles of different sizes depositing in different regions of the respiratory tract. ${ }^{6}$ Particles in the size range 2-5 $\mu \mathrm{m}$ are optimal for delivering drugs into the central regions of the lung and hence pMDI are formulated with micronized drug particles in this size range.

If the inhaled particles are hygroscopic then their water content and hence size and aerodynamic properties are dependent upon the environmental relative humidity $(\mathrm{RH})$ and the trajectory of the aerosol particles though this environment. The RH environment of a particle exiting the pMDI has three distinct regions: (1) completely dry at the initial point of injection as the aerosol is released from the canister, (2) at ambient RH as the patient inhales both the aerosol and the ambient atmosphere, and finally (3) high RH as the drug particles enters into the wet walled respiratory tract. The change in RH from phase (2) to (3) is not monotonic. As the particle travels deeper into the respiratory system it encounters increasingly higher humidities: $\sim 40 \%$ in the mouth, $\sim 60 \%$ in 
the pharynx, to finally approaching near water saturation $\sim 99.5 \%$ in the deep airways. ${ }^{6}$ The timescale of inhalation, and particle impaction, is $\sim 10 \mathrm{~s}$. Patients using pMDIs are advised to actuate the device whilst simultaneously inhaling slowly and steadily for $4 \mathrm{~s}$, followed by a breath-hold of $10 \mathrm{~s}$ to promote deposition by gravitational sedimentation. ${ }^{7}$

Hygroscopic particles moving from a region of low ambient humidity into one of high humidity would be expected to increase in size due to water uptake. A change in particle size will influence the site of particle deposition in the lung following inhalation. ${ }^{8}$ A particle predicted to deposit in the central regions of the lung may, if substantial growth is experienced, deposit higher in the respiratory tract than expected, potentially resulting in reduced therapeutic efficacy and increased incidence of side effects (e.g. oral candidiasis following deposition of corticosteroids in the oropharynx). To mitigate these negative implications, the hygroscopic properties of respirable drug formulations need to be understood. In particular the kinetics of deliquescence and particle growth need to be measured over the timescale ( $\sim 10 \mathrm{~s})$ of the journey of the aerosol from pMDI to lung. The kinetics could be significantly influenced by the morphology, size and/or phase of the particle and thus investigating them for the real pMDI-generated, contact-free particle is essential to obtain realistic results. Previous studies have investigated the hygroscopicity of aqueous formulations of respirable drugs generated by nebulizer e.g. Haddrell et al. 2014. ${ }^{9}$ To the best of our knowledge this is the first in situ study of pMDI generated particles which represent the dominant commercial methodology for transporting aerosols into the lungs.

The optical trap used in this study utilized a new design of counter-propagating dual beam (CPDB) configuration which we recently reported. ${ }^{10,11}$ The trap is optimized to trap solid micron sized particles over long timescales. This optical trapping configuration can readily capture non-spherical aerosol particles. The counter-propagating trapping beams are generated from the output of a Nd:Yag laser operating at $1064 \mathrm{~nm}$ (Ventus, Laser Quantum).

Typical laser powers used are $14 \mathrm{~mW}$ through the top and $10 \mathrm{~mW}$ through the bottom objective lenses (see Fig. 1). To achieve stable

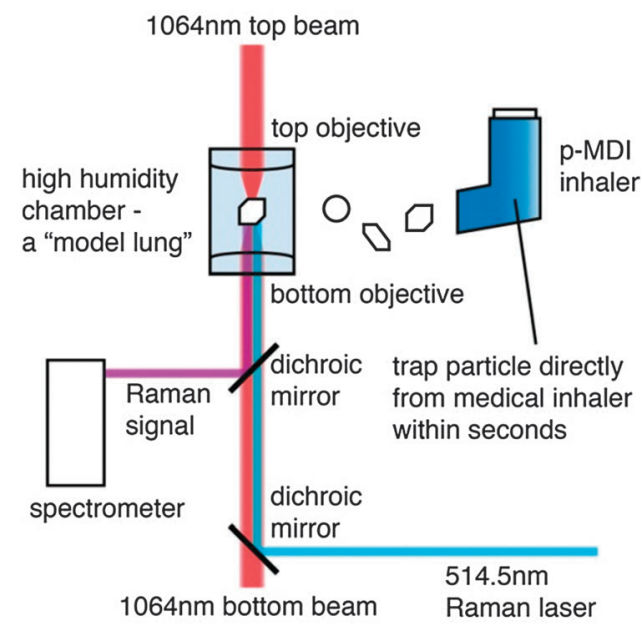

Fig. 1 Schematic cartoon of the experimental setup showing the trapping and Raman laser paths and high humidity particle trap. trapping the focal separation between the top and bottom beams was adjusted to $\sim 10 \mu \mathrm{m}$ which allowed for stable levitation of the same particle for long time periods ( $>1$ hour). Most experiments were carried out over shorter, medically relevant timescales.

The pMDI inhaler is discharged under ambient laboratory relative humidity ( $c a .30 \%$ ) and is directed into a small bespoke environment chamber, using a $20 \mathrm{~cm}$ length of Teflon tubing (6 $\mathrm{mm}$ internal diameter). Inside the environmental chamber the relative humidity can be varied, and very high subsaturated relative humidities ( $>98 \%$ ) can be obtained.

The experiments were performed at ambient laboratory temperature $c a .20{ }^{\circ} \mathrm{C}$. Within the chamber the humidity and temperature were measured using a probe (Sensirion SHT75) positioned within $\sim 1 \mathrm{~cm}$ of the trapping position. High humidity conditions were achieved using a water reservoir within the chamber. Lower humidity conditions were generated by flowing dry nitrogen, generated from liquid nitrogen boil-off, through the chamber using a flow controller (Bronkhorst MV-301) with flow rates up to $200 \mathrm{sccm}$. The time taken from particle release from pMDI to levitation within the optical trap was on the order of seconds and hence the chamber could closely replicate the residence time and the $\mathrm{RH}$ trajectory of the particle in the lung.

Aqueous droplets of SS were generated from a $0.05 \mathrm{M}$ solution of SS (99\% Alfa Aesar) in MilliQ water using an ultrasonic nebulizer (Schill Mobil Aerosonic 3060). No attempt was made to replicate the minute amounts of ethanol cosolvents present in the Salamol ${ }^{\mathbb{R}}$ pMDI formulation and the role of ethanol is assumed to be negligible. The solution generated droplets were trapped within the CPDB trap in the diameter size range 1-2 $\mu \mathrm{m}$.

To obtain spectroscopic information regarding particle composition a $514.5 \mathrm{~nm}$ Ar-ion laser (Innova 308C, Coherent) was focused onto the trapped particle through the lower objective with a power of $2 \mathrm{~mW}$ at focus (see Fig. 1). This light source was used to generate both Raman and fluorescent (if present) signal which provide compositional information about the probed particle. Both the Raman and fluorescent signal were directed to a spectrograph (Spectrapro 2500i, 1200 groove blazed at $500 \mathrm{~nm}$ ) and imaged onto a CCD camera (Princeton Instruments, Spec10). Each particle was only subjected to the $514.5 \mathrm{~nm}$ laser for $30 \mathrm{~s}$ to minimize heating and fluorescent bleaching effects. ${ }^{12}$ This necessitates using fresh particles for every measurement. In addition to spectroscopic interrogation the trapped particles/droplets were imaged using brightfield microscopy. For more detail on the optical strategy, see Rkiouak et al. $2014 .^{10}$

Optical trapping of particles can lead to particle heating through absorption of the laser radiation incident on the particle. The extent of the heating depends upon the characteristics of the laser beam (wavelength, power and beam profile) and the absorption characteristics of the particle and surrounding medium. ${ }^{13,14}$ The increase in particle temperature above the ambient conditions, caused by absorption of the 514.5 and $1064 \mathrm{~nm}$ laser beams, is estimated using an approach similar to Knox and Reid. ${ }^{15}$

At the typically used laser powers it is estimated that a pure salbutamol sulphate particle of $2 \mu \mathrm{m}$ diameter would be $1.4{ }^{\circ} \mathrm{C}$ warmer than the ambient conditions (see ESI $\dagger$ ).

The optical trap configuration was extremely successful at trapping solid pMDI generated particles over a range of particle 
sizes and shapes. The trapped particle size ranged from $c a$. 1-3 $\mu \mathrm{m}$. The measured asymmetry factor corresponded to particle shapes ranging from spheres to rods (Fig. 2). The trap only ever contains one particle at a time as observed through bright field microscopy. Panel A in Fig. 2 provides representative brightfield microscopy images of levitated pMDI particles with asymmetry increasing from left to right. The brightfield images of SS nebulised droplets and SS particles at high humidity both display more intense illumination at the centre of the particle, typical for the illumination of microspheres. Dry particles do not display this characteristic.

Panel B compares the particle size histograms of particles caught within the optical trap and the total particle output from the pMDI. The C panels compare the sphericity of optically trapped particles, under high and low humidity conditions, with the total pMDI output obtained by imaging a coverslip at ambient RH.

The size and sphericity of the total output from the pMDI is measured by releasing several puffs of the pMDI into the environmental chamber, without the optical trap active, and letting the particles settle onto the coverslip through gravitational sedimentation. The sizes and shapes of the resulting particle ensemble are then imaged using the brightfield microscope. Particle size and shape were analysed using the image analysis software Image (release 1.48) with particle size defined by the longest axis of the particle, and the particle sphericity defined by the reciprocal of the aspect ratio. The measured particle size was calibrated using a graticule placed on the microscope.

In Fig. 2 panel B, the optical trap captures particles that are representative of the full output size range of the pMDI. The modal size for both the levitated particles and full inhaler output is $\sim 2 \mu \mathrm{m}$. However, the optical trap's particle size distribution is narrower. It does not stably capture the smallest $(<1 \mu \mathrm{m})$ or the larger particles $(>3.5 \mu \mathrm{m})$ that are observed in the full output of the pMDI. The observed trapped particle sizes and shapes were consistent with the measured aerosol output of pMDI from previous studies. ${ }^{16}$
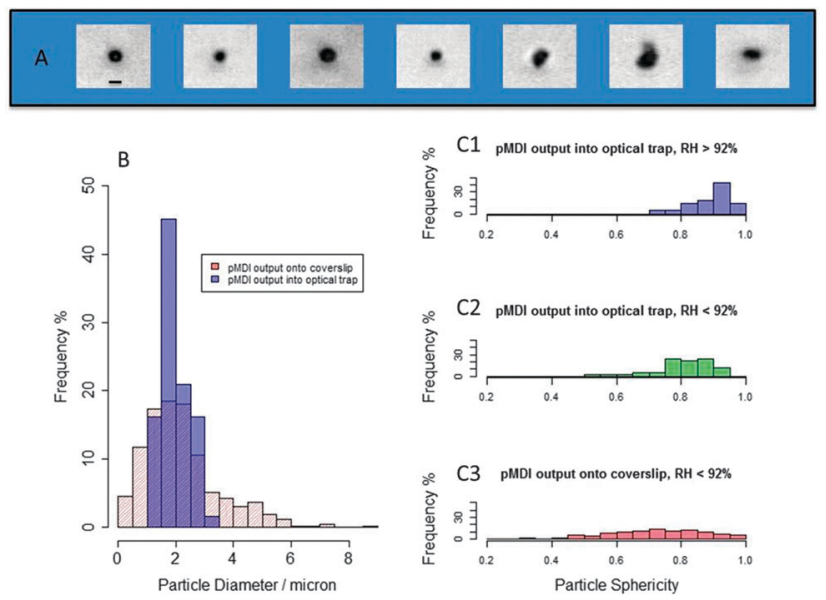

Fig. 2 Imaging, characterising and comparing levitated particle with total pMDI output on coverslip. Panel A - example images of levitated SS particles with sphericity decreasing from left to right (scale bar $=2 \mu \mathrm{m}$ ). Panel $B-$ histograms of particle diameter (major axis). Panel C - histograms of particle sphericity caught in the optical trap under low (C1) and high (C2) $\mathrm{RH}$ conditions, and output collected on cover slip (C3). The histograms are constructed from data from 62 optically levitated particles and 534 particles on the coverslip.
Under low $\mathrm{RH}$ conditions, the particles caught in the optical trap tend to be more spherical than the average particle exiting the pMDI (Fig. 2C2 vs. Fig. 2C3). This is likely due to the optical trap requiring some degree of symmetry in the particle shape for efficient trapping. The sphericity of the trapped particles increases significantly under high humidity conditions, suggesting that water uptake by the particles increases particle sphericity (Fig. 2C1). No increase in average size was observed between low and high $\mathrm{RH}$ particles because the trap is optimized to a certain size range $(\sim 1-3 \mu \mathrm{m})$.

The chemical and physical properties of the SS aerosol particle were followed using optical imaging, Raman spectroscopy and fluorescence spectroscopy. Raman spectra of optically trapped pMDI generated particles were measured over a wide range of RH (10-98\%). The spectra of pMDI particles were compared to the spectra from both bulk crystalline SS material deposited on a coverslip and optically trapped aqueous droplets of SS generated through nebulization (Fig. 3). The Raman spectrum of the crystalline SS from this study is similar to that previously recorded by Ali et al. but the spectral resolution in this study is greater. ${ }^{17}$ The four largest intensity peaks at 780, 978, 1255 and 1615 rel. $\mathrm{cm}^{-1}$ are assigned to $\nu(\mathrm{C}-\mathrm{C}-\mathrm{O}), \nu(\mathrm{COH})$, $\delta(\mathrm{CH})$ and hydrogen bonded $\mathrm{C}-\mathrm{OH}$, respectively. ${ }^{17}$ The spectra of SS droplets generated from nebulization of aqueous SS contain a strong fluorescence signal superimposed upon the SS Raman peaks which are significantly broadened compared to the crystalline sample. The Raman peak broadening in solution is likely due to the increase in vibrational energy configurations of the SS once water molecules are available for interaction via hydrogen bonding. Previous studies have also observed the fluorescence of solution phase SS. ${ }^{18,19}$

The pMDI generated particles show two distinct spectra which are dependent upon $\mathrm{RH}$. At $\mathrm{RH}$ below $\sim 92 \%$ the spectra are nearidentical to the bulk crystalline material, confirming that the particles generated by the pMDI are largely crystalline in composition as they exit the pMDI. The particles caught in the chamber at $\mathrm{RH}$ greater than $\sim 92 \%$ show similar spectra but with significant peak broadening similar to the nebulised SS droplets. All four major peaks (780, 978, 1255 and 1615 rel. $\mathrm{cm}^{-1}$ ) and the 750 rel. $\mathrm{cm}^{-1}$ peak show significant broadening at elevated RH, as shown in Fig. 3. There is also significant growth in the peak at $1450 \mathrm{rel} . \mathrm{cm}^{-1}$.

We attribute the step change in peak broadening at 92\% $\mathrm{RH}$ to a previously unreported SS deliquescent phase transition. We note that amorphous SS particles have a distinct Raman spectrum ${ }^{20}$ and therefore the transformation at $c a .92 \% \mathrm{RH}$ is unlikely to be caused by a crystalline to amorphous phase transition.

To further investigate the phase transition we looked at the peak broadening in more detail. In particular, we investigated the 750 and 780 rel. $\mathrm{cm}^{-1}$ peaks. These peaks were chosen because they reside in a spectrally simple region and can be successfully fitted with two Lorentzian profiles with low associated error. The results of the 780 rel. $\mathrm{cm}^{-1}$ peak fitting are shown in the right panel of Fig. 3. There is a large and obvious change in the peak width of the $780 \mathrm{~cm}^{-1}$ band after deliquescence. Before deliquescence the average full width at half maximum (FWHM) is $6.5 \pm 0.7 \mathrm{rel} . \mathrm{cm}^{-1}$ and after deliquescence the width is $28.3 \pm 1.8 \mathrm{rel} . \mathrm{cm}^{-1}$, where the errors are $1 \sigma$. The FWHM width of $750 \mathrm{~cm}^{-1}$ peak also changes significantly upon deliquescence with the pre-deliquescence and post-deliquescence values equal to $6.0 \pm 0.9 \mathrm{rel} . \mathrm{cm}^{-1}$ 

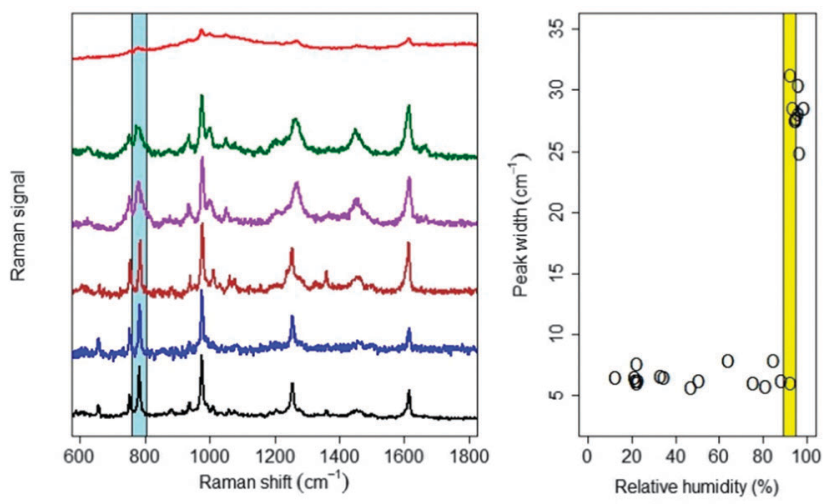

Fig. 3 Left panel: offset Raman spectra of SS. Black line - Raman signal of crystalline SS (Alfa Aesar) deposited on the cover slip. Blue, brown, purple and green line - Raman signal from a single SS particle injected from the pMDI (Salamol ${ }^{\circledR}$ ) into the environmental chamber and optically trapped at $12,92,94$ and $98 \% \mathrm{RH}$, respectively. Red line - Raman and fluorescence signal from single SS droplet generated by nebulization of aqueous SS solution. Right panel: width (FWHM of fitted Lorentzian function) of the peak at $780 \mathrm{~cm}^{-1}$, which is highlighted by the blue band in the left panel. The yellow band indicates the SS deliquescence point.

and $11.6 \pm 1.4$ rel. $\mathrm{cm}^{-1}$, respectively. The peak broadening analysis determines the $\mathrm{RH}$ value at which the deliquescence phase transition occurs: $91.9 \pm 2.7 \%$. The error is dominated by the $\mathrm{RH}$ probe accuracy as stated by the manufacturer.

Simple peak broadening analysis on the other three major peaks $\left(978,1255,1615\right.$ rel. $\left.\mathrm{cm}^{-1}\right)$ give qualitatively very similar results with a clear transition occurring at $c a .92 \%$ RH. Notably, the phase transition is possibly only partial on the timescale of the experiment, as evidenced by the lack of complete particle sphericity in all particles (Fig. 2C1). However, the lack of sphericity could also be largely explained by error in the particle size measurement. ${ }^{21}$

Interestingly, no fluorescence signal was observed from the levitated particle at $\mathrm{RH}$ either above or below the deliquescence point. However, fluorescence is highly sensitive to the presence and concentration of solvents as well as $\mathrm{pH}^{22}$ A polar solvent like water can lead to lower energy fluorescence emission at longer wavelengths. ${ }^{22}$ Hence we would expect the fluorescence properties of nebulized droplets to be distinct from particles generated by a pMDI regardless of the RH due to the higher water content of nebulized particles.

We provide a new methodology to study the physical and chemical characteristics of SS aerosol directly as it exits from a commercially available pMDI without changing its physicalchemical properties. Furthermore we interrogate the changes in aerosol characteristics that occur once the aerosol enters into a purpose built high humidity environment chamber which is used as a proxy for the human respiratory tract. This study focused on the output from a Salamol ${ }^{\mathbb{R}}$ inhaler, but the technique presented should be universal for all pMDI devices. SS particles are observed to deliquesce at $91.9 \pm 2.7 \% \mathrm{RH}$ as evidenced by three separate pieces of information: significant peak broadening in the Raman spectrum, increased particle sphericity and the emergence of a central bright spot within the brightfield images which is indicative of droplets. Thus, following release from the Salamol ${ }^{\circledR}$ pMDI into the high $\mathrm{RH}$ environment of the respiratory tract, crystalline SS takes up water via deliquescence, resulting in an increase in particle sphericity. This change in particle size, phase and morphology will affect the aerodynamic properties of the aerosol, and should be considered when modelling the aerosolization performance of inhalation products. In addition, exposure to high $\mathrm{RH}$ results in the change of crystalline to liquid phase which will almost certainly promote drug dissolution, and hence the kinetics of drug uptake, following the deposition of the droplet onto the epithelial lining of the respiratory tract. The reported experiments were performed at a temperature lower than the human body and at RH slightly less than saturation. Future work will focus on more realistic proxies for the respiratory tract.

This work was conducted at the STFC Central Laser Facility based at the Research Complex at Harwell, and was supported through STFC programme access scheme (application No.: 13230009). We thank NERC (NE/J500070/1, NE/I528277/1) and ERC (279405) for financial support. We thank Nicholas Davidson for providing the UV-Vis-NIR spectrum of SS.

\section{Notes and references}

1 M. King, K. C. Thompson and A. D. Ward, J. Am. Chem. Soc., 2004, 126(51), 16710-16711.

2 British National Formulary, BMJ Group and Pharmaceutical Press, London, 66th edn, 2014.

3 F. Lavorini, C. J. Corrigan, P. J. Barnes, P. R. Dekhuijzen, M. L. Levy, S. Pedersen, N. Roche, W. Vincken and G. K. Crompton, Respir. Med., 2011, 105(7), 1099-1103.

4 B. Aggarwal and J. Gogtay, Expert Rev. Respir. Med., 2014, 8(3), 349-356.

5 J. Bell and S. Newman, Expert Opin. Drug Delivery, 2007, 4(3), 215-234.

6 D. M. Broday and P. G. Georgopoulos, Aerosol Sci. Technol., 2001, 34, 144-159.

7 G. Taylor and I. Kellaway, Pulmonary Drug Delivery, in Drug Delivery and Targeting for Pharmacists and Pharmaceutical Scientists, ed. A. M. Hillery, A. W. Lloyd and J. Swarbrick, CRC Press, London, 2001.

8 N. R. Labiris and M. B. Dolovich, Br. J. Clin. Pharmacol., 2003, 56(6), 588-599.

9 A. E. Haddrell, J. F. Davies, R. E. H. Miles, J. P. Reid, L. A. Dailey and D. Murnane, Int. J. Pharm., 2014, 463, 50-61.

10 L. Rkiouak, M. J. Tang, J. C. J. Camp, J. McGregor, I. M. Watson, R. A. Cox, M. Kalberer, A. D. Ward and F. D. Pope, Phys. Chem. Chem. Phys., 2014, 16, 11426-11434.

11 M. J. Tang, J. C. J. Camp, L. Rkiouak, J. McGregor, I. M. Watson, R. A. Cox, M. Kalberer, A. D. Ward and F. D. Pope, J. Phys. Chem. A, 2014, 118(38), 8817-8827.

12 H. Wang, M. A. Boraey, L. Williams, D. Lechuga-Ballesteros and R. Vehring, Int. J. Pharm., 2014, 469, 197-205.

13 E. J. G. Peterman, F. Gittes and C. F. Schmidt, Biophys. J., 2003, 84, $1308-1316$

14 H. Mao, J. R. Arias-Gonzalez, S. B. Smith, I. Tinoco and C. Bustamante, Biophys. J., 2005, 89, 1308-1316.

15 K. J. Knox and J. P. Reid, J. Phys. Chem. Lett., 2008, 1123, 10439-10441.

16 C. Dunbar and J. Mitchell, J. Aerosol Med., 2005, 18(4), 439-451.

17 H. R. H. Ali, H. G. M. Edwards, J. Kendrick and I. J. Scowen, Drug Test. Anal., 2009, 1, 51-56.

18 H. N. Pandya, H. H. Berawala, D. M. Khatri and P. J. Mehta, Pharma. Methods, 2010, 1(1), 49-53.

19 L. G. Dodson, R. A. Vogt, Y. Marks, C. Reichardt and C. E. CrespoHernández, Chemosphere, 2011, 83, 1513-1523.

20 P. N. Balani, S. Y. Wong, W. K. Ng, E. Widjaja, R. B. H. Tan and S. Y. Chan, Int. J. Pharm., 2010, 391, 125-136.

21 The size error in the brightfield imaging is $0.2 \mu \mathrm{m}$ as defined by the pixel size on the imaging camera. For particles $1-3 \mu \mathrm{m}$ in size this leads to a possible error in the calculated sphericity of approximately $7-20 \%$.

22 J. R. Lakowicz, Principles of Fluorescence Spectroscopy, Springer, 3rd edn, 2006. 